\title{
Innovative Data Fusion Enabled Structural Health Monitoring Approach
}

\author{
Xiukuan Zhao, ${ }^{1,2}$ Ruolin Wang, ${ }^{3}$ Haichang Gu, ${ }^{4}$ Gangbing Song, ${ }^{4,5}$ and Y. L. Mo ${ }^{6}$ \\ ${ }^{1}$ Key Laboratory of Ionospheric Environment, Institute of Geology and Geophysics, Chinese Academy of Sciences, \\ Beijing 100029, China \\ ${ }^{2}$ Beijing National Observatory of Space Environment, Institute of Geology and Geophysics, Chinese Academy of Sciences, \\ Beijing 100029, China \\ ${ }^{3}$ School of Civil Engineering, Wuhan University, Wuhan, Hubei 430072, China \\ ${ }^{4}$ Department of Mechanical Engineering, University of Houston, Houston, TX 77204, USA \\ ${ }^{5}$ School of Civil Engineering, Dalian University of Technology, Dalian, Liaoning 116024, China \\ ${ }^{6}$ Department of Civil and Environmental Engineering, University of Houston, Houston, TX 77204, USA
}

Correspondence should be addressed to Ruolin Wang; rl.wang@whu.edu.cn

Received 10 October 2013; Accepted 23 December 2013; Published 19 February 2014

Academic Editor: Xiao-Wei Ye

Copyright (C) 2014 Xiukuan Zhao et al. This is an open access article distributed under the Creative Commons Attribution License, which permits unrestricted use, distribution, and reproduction in any medium, provided the original work is properly cited.

Piezoceramic-based active sensing is a useful approach to structural health monitoring. This approach often involves a large number of distributed piezoceramic transducers. It may be confusing to incorporate each sensor data. It is desired to develop an automated health monitoring approach to obtain a comprehensive and accurate health monitoring result by simultaneously interpreting data from all sensors. In this paper, an innovative data fusion enabled structural health monitoring (SHM) approach based on the Dempster-Shafer (D-S) evidence theory is proposed to obtain comprehensive SHM results for a distributed sensor network in a civil infrastructure. Considering that evidence from multiple different information sources (sensor data) has different levels of significance, not all evidence is equivalently effective for the final decision. A weighted fusion damage index (WFDI) is proposed to perform damage identification based on the authors' recently developed piezoceramic-based smart aggregates. Experimental data of a two-story concrete frame was used to study the effectiveness of the proposed weighted fusion damage index. Analyses show that the proposed weighted fusion damage index can reveal the damage status of different areas of the frame. The results are consistent with the visual inspection of the cracks on the concrete frame.

\section{Introduction}

Health monitoring of civil infrastructures has received considerable attention in recent years. Many events, such as impacts, earthquakes, or hurricanes, cause damage to structures. It is important to provide accurate and comprehensive health monitoring results after such an event to decisionmakers. Using piezoelectric transducers, there are many health monitoring approaches, such as the electromechanical (E/M) impedance method (Soh et al. [1], Naidu and Soh [2], $\mathrm{Fu}$ and $\mathrm{Xu}$ [3], and Kral et al. [4]) and the active-sensing method (Ihn and Chang [5], Song et al. [6, 7], Laskar et al. [8], and $\mathrm{Yu}$ et al. [9]). Often multiple sensors are employed in a structural health monitoring (SHM) system [10-12]. The interpretation of health monitoring data from each individual sensor may be confusing and may not lead to accurate structural health status. It is desired to develop an automated approach to obtain final comprehensive structural health status based on multiple sensors (information sources). In this paper, data fusion technique is proposed to process the data obtained from multiple piezoceramic transducers in a complex civil structure to provide an accurate and comprehensive health monitoring result.

Data fusion combines and processes data from multiple information sources and related information from associated databases to achieve improved accuracies and more specific inferences than the results derived by the use of a single source alone. It has attracted increasing attention in structural 
health monitoring due to its inherent capabilities in extracting information from different sources and integrating them into a consistent, accurate, and intelligible dataset. Some researchers studied damage identification methods using data fusion techniques to improve accuracy and obtain more specific inferences. Guo and Zhang [13] and Guo [14] regarded the changes of frequencies and mode shapes as two different information sources and used the data fusion method to detect the damage of two-dimensional truss structures. Three main fusion approaches were studied in this research. Vanniamparambil et al. [15] proposed a novelty detector based on the Mahalanobis distance which was implemented in a data fusion scheme to assess the extent of damage. Their results proved the effectiveness by combining real-time optical and acoustic nondestructive testing, while Bao and $\mathrm{Li}$ [16] employed the Dempster-Shafer (D-S) evidence theory and Shannon entropy to decrease the uncertainty and improve the accuracy of damage identification. In addition, data fusion technology is improved by integrating with artificial intelligence, such as fuzzy logic and genetic algorithms. Fan and Zuo [17] improved the D-S theory through the introduction of a fuzzy membership function, importance index, and conflict factor in order to address the issues of evidence sufficiency, evidence importance, and conflicting evidence in the practical application of the D-S evidence theory. Guo and Li [18] presented a two-stage method in determining the location and extent of multiple structural damages by using the information fusion technique and genetic algorithm. Thenozhi et al. [19] used numerical integrator and convexconcave hull classification for structural health monitoring of tall buildings. Zhao et al. [20] introduced a hierarchical ensemble scheme to the data fusion field. Health monitoring of a small-scale two-story frame structure with different types of damage subject to shaking table tests was used as an example to validate the efficiency of their proposed scheme.

Piezoceramic-based active sensing is a useful approach to structural health monitoring. This approach often involves a large number of distributed piezoceramic transducers. It may be confusing to incorporate each sensor data. It is desired to develop an automated health monitoring approach to obtain a final, comprehensive, and accurate health monitoring result by simultaneously interpreting data from all sensors. In this paper, an innovative data fusion enabled structural health monitoring (SHM) approach based on the Dempster-Shafer (D-S) evidence theory is proposed to obtain comprehensive SHM results for a distributed sensor network in a civil infrastructure. Considering that evidence from multiple different information sources (sensor data) has different levels of significance, not all evidence is equivalently effective for the final decision. Different important levels were considered for evidence by assigning weighted coefficients. A weighted fusion damage index (WFDI) is proposed to perform damage identification based on the authors' recently developed piezoceramic-based smart aggregates. Experimental data of a two-story concrete frame was used to study the effectiveness of the proposed weighted fusion damage index. The results indicate that the proposed method can improve damage identification accuracy and increase the reliability of damage identification.

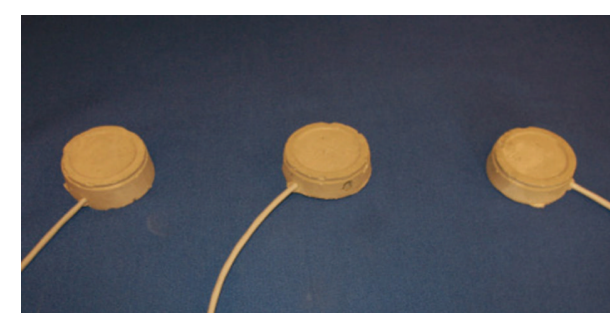

FIgURE 1: Three fabricated smart aggregates.

In this paper, data fusion is used to process structural health monitoring data from multiple sensors to provide an accurate and final interpretation of the structural health status. In the proposed approach, based on the D-S evidence theory, a weighted fusion damage index is proposed by comparing the transmission energy difference between the signatures of healthy and damaged states. To verify the effectiveness of the proposed innovative approach, the data fusion method was used to analyze the experimental data obtained in a structural push-over test of a two-story concrete frame. These experimental data were obtained through an active structural health monitoring approach using piezoceramicbased smart aggregates. Analyses show that the proposed weighted fusion damage index can reveal the damage status of different areas of the frame. The results are consistent with the visual inspection of the cracks on the concrete frame, which indicate that the proposed method can provide an accurate and comprehensive interpretation of the structural health status by using data from multiple sensors.

\section{Active Structural Health Monitoring Using Embedded Smart Aggregates}

In this study, experimental health monitoring data were obtained by smart aggregates in active-sensing tests and then analyzed by data fusion method to get results with improved accuracy. The developed smart aggregate can either be used as an actuator to excite stress waves to propagate through concrete structure or used as a sensor to detect the wave response.

2.1. Piezoceramic-Based Smart Aggregate. The developed piezoceramic-based smart aggregates are low cost, multifunctional devices which are capable of performing early-age strength monitoring, impact detection, and structural health monitoring for concrete structures [7]. PZT (lead zirconate titanate), a type of piezoceramic material with strong piezoelectric effect, is used to develop the smart aggregates. Smart aggregates (SAs), as shown in Figure 1, are formed by embedding a waterproof PZT patch with lead wires, as shown in Figure 2, into a small concrete block before installing smart aggregates into a larger concrete structure. The materials used to build the small concrete block have the same mixture ratio of cement, sand, and water as the host concrete structure. This way, the smart aggregates will have almost no effect in changing the material and structural properties of the host concrete structures. 


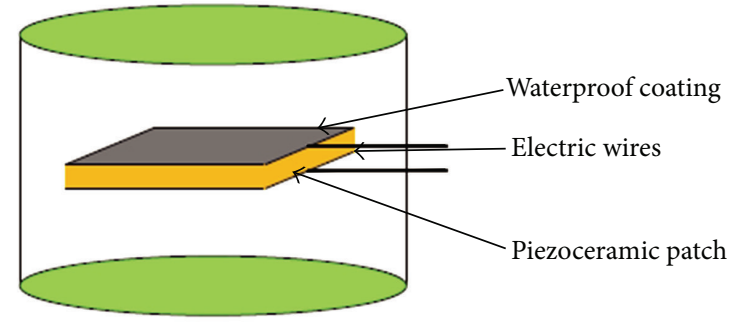

FIGURE 2: Illustration of a smart aggregate.



FIgURE 3: Active sensing enabled by smart aggregates.

For concrete structural health monitoring, a smart aggregate enabled active-sensing system was developed as illustrated in Figure 3. In the proposed active-sensing system, one smart aggregate is used as an actuator to generate a sweep sine signal, while the other smart aggregates are used as sensors to detect the sweep sine responses. The crack or damage inside the concrete structure acts as stress relief in the wave propagation path. The amplitude of the wave and the transmission energy will decrease due to the existence of the crack. The drop value of the transmission energy will be correlated with the degree of the damage inside. In this paper, PZTA stands for the smart aggregate used as an actuator and PZTS stands for the smart aggregate used as a sensor.

2.2. Root-Mean-Square Deviation and Damage Index. Various kinds of damage indices have been developed for health monitoring of civil structures in recent years [21, 22]. The root-mean-square deviation (RMSD) is commonly used as a damage index to compare the difference between the signatures of healthy and damaged states.

In this research, wavelet packet analysis is used as a signal-processing tool to analyze the sensor signal of the embedded PZT patches in the concrete structure. The advantage of wavelet packet analysis is that it enables the inspection of relatively narrow frequency bands over a relatively shorttime window. The sensor signal $S$ is decomposed by an $n$-level wavelet packet decomposition into $2^{n}$ signal sets $\left\{X_{1}, X_{2}, \ldots, X_{2^{n}}\right\} . X_{j}$ can be expressed as $X_{j}=\left[x_{j, 1}, x_{j, 2}, \ldots\right.$, $\left.x_{j, m}\right]$, where $j$ is the frequency band $\left(j=1, \ldots, 2^{n}\right)$ and $m$ is the amount of sampling data. The energy of the decomposed signal is defined as $E_{i, j}=\left\|X_{j}\right\|_{2}^{2}=x_{j, 1}^{2}+x_{j, 2}^{2}+\cdots+x_{j, m}^{2}$, where $i$ is the time index and $j$ is the frequency band $\left(j=1, \ldots, 2^{n}\right)$. The energy vector at time index $i$ is defined as $E_{i}=\left[E_{i, 1}\right.$, $\left.E_{i, 2}, \ldots, E_{i, 2^{n}}\right]$.

In the proposed approach, the damage index is formed by calculating the RMSD between the energy vectors of the healthy state and the damaged state. The energy vector for healthy data is $E_{h}=\left[E_{h, 1}, E_{h, 2}, \ldots, E_{h, 2^{n}}\right]$, and the energy vector $E_{i}$ for the damaged state at time index $i$ is defined as $E_{i}=\left[E_{i, 1}, E_{i, 2}, \ldots, E_{i, 2^{n}}\right]$. The damage index at time $i$ is defined as

$$
I_{i}=\sqrt{\frac{\sum_{j=1}^{2^{n}}\left(E_{i, j}-E_{h, j}\right)^{2}}{\sum_{j=1}^{2^{n}}\left(E_{h, j}\right)^{2}}} .
$$

The proposed damage index represents the transmission energy loss portion caused by structural damage. When the damage index is close to 0 , the concrete structure is in a healthy state. However, when the damage index is larger than a certain threshold value, damage begins to appear in the concrete structure. The proposed damage index is used to quantitatively evaluate the damage severity. The greater the index is, the more severe the damage that emerges is.

\section{Weighted Fusion Damage Index (WFDI)}

3.1. Dempster-Shafer Evidence Theory. The Dempster-Shafer (D-S) theory is a mathematical theory of evidence, introduced in the 1960s by Dempster [23] and developed in the 1970s by Shafer [24]. Yager and Liu [25] summarized the classic works of the Dempster-Shafer theory, and the evidence theory is briefly summarized as follows.

For a finite set of mutually exclusive and exhaustive propositions, $\Theta$ is called a frame of discernment, where $2^{\Theta}$ is the set of all the subsets of $\Theta: 2^{\Theta}=\{A \mid A \subseteq \Theta$. The subset $A$ includes two special cases, which are the null set $\phi$ and the full set $\Theta$.

The evidence theory allows the basic probability assignment (BPA) to individual propositions and also to any subsets of the power set provided that the sum of all basic probability is equal to one:

$$
m(\phi)=0, \quad \sum_{A \subseteq \Theta} m(A)=1, \quad \Theta=\left\{A_{i} \mid i=1,2, \ldots, N\right\} .
$$

The belief function (Bel) is a belief measure of proposition $A$, and it sums the mass value of all the nonempty subsets of $A$ as

$$
\operatorname{Bel}(A)=\sum_{B \subseteq A} m(B)
$$

The plausibility function $(\mathrm{Pl})$ takes into account all the elements related to $A$ as

$$
\operatorname{Pl}(A)=\sum_{B \cap A \neq \emptyset} m(B) .
$$




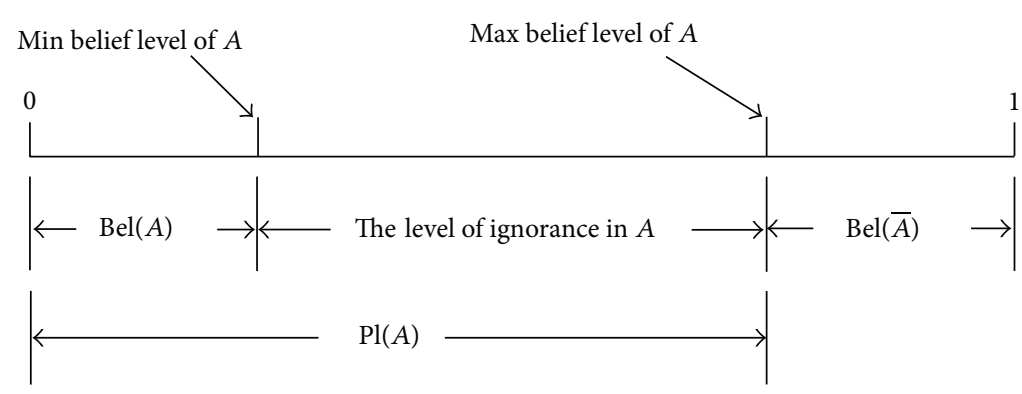

FIgUre 4: Relationship between $\operatorname{Bel}(A)$ and $\operatorname{Pl}(A)$.

The belief function represents the lower limit of the probability, and the plausibility function provides the upper limit of the probability. $[\operatorname{Bel}(A), \mathrm{Pl}(A)]$ is the confidence interval which describes the uncertainty of $A$. The relationships between Bel value, $\mathrm{Pl}$ value, and uncertainty are described in Figure 4.

Multiple evidence can be fused using Dempster's combination rule, which is also called the orthogonal sum of evidence, as shown below:

$$
\begin{gathered}
\left(m_{1} \oplus \cdots \oplus m_{n}\right)(A)=\frac{1}{K} \sum_{A_{1} \cap \cdots \cap A_{n}=A} m_{1}\left(A_{1}\right) \cdots m_{n}\left(A_{n}\right), \\
K=\sum_{A_{1} \cap \cdots \cap A_{n} \neq \emptyset} m_{1}\left(A_{1}\right) \cdots m_{n}\left(A_{n}\right) \\
=1-\sum_{A_{1} \cap \cdots \cap A_{n}=\emptyset} m_{1}\left(A_{1}\right) \cdots m_{n}\left(A_{n}\right) .
\end{gathered}
$$

3.2. Weighted Fusion Damage Index. To fuse the information of the same location sensor received from different actuators, each piece of the information received from different actuators can be considered as a piece of evidence for that sensor. In reality, evidence has varying degrees of importance. The conventional D-S evidence theory does not differentiate this. In structural health monitoring, since different evidence makes different contributions to different damage detection, evidence importance should be considered for specific damage detection.

In the piezoceramic-based active-sensing system for structural health monitoring, consider one PZTS (the PZT transducer is utilized as a sensor) data received from different PZTAs (the PZT transducer is utilized as an actuator) as the multidata in data fusion. The structure has two statuses: failure $\left(F_{1}\right)$ and healthy $\left(F_{2}\right)$. The full set is $\Theta=\left\{F_{1}, F_{2}\right\}$. The original BPA can be obtained from the damage index, $m_{i, j}\left(F_{1}\right)=I_{i, j}, m_{i, j}\left(F_{2}\right)=1-I_{i, j}, m_{i, j}(\Theta)=0$, where $i$ is the $i$ th PZTS and $j$ is the $j$ th PZTA.

Since the PZTS has different distances between different PZTAs, the contributions of the data from different PZTAs for specific damage detection are also different. To fuse the data of PZTS from different PZTAs, the importance index of the evidence from $\operatorname{PZTA}(j)$ can be written as $v_{j}$, which can be obtained by the distance between PZTS and PZTA $(j)$ :

$$
v_{j}=\frac{1}{d_{j}}
$$

where $d_{j}$ is the distance between PZTS and PZTA $(j)$. Compared with other factors, the distance is more important in extracting the damage location information and evaluating the severity of damage. The smaller the distance between an actuator-sensor pair is, the more important the data of this actuator-sensor pair is in providing the location information and quantitatively evaluating damage. In Section 4.2, we will show how the distance $d_{j}$ influences the evaluation of the structural health status.

For each PZTS, the largest important index will be taken as the denominator. The weighted index of BPA for PZTS, denoted by $w_{j}$, can be expressed as

$$
w_{j}=\frac{v_{j}}{\max \left\{v_{j} \mid j=1,2, \ldots, n\right\}} .
$$

The matrix $W$ of the weighted index for each PZTS can be obtained by using (7) as

$$
W=\begin{gathered}
E_{1} \\
E_{2} \\
\vdots \\
E_{n}
\end{gathered} \mid\left[\begin{array}{ccc}
\left.F_{1}\right\} & \left\{F_{2}\right\} & \Theta \\
w_{1} & w_{1} & w_{1} \\
w_{2} & w_{2} & w_{2} \\
\vdots & & \\
w_{n} & w_{n} & w_{n}
\end{array}\right],
$$

where $E_{j}(j=1, \ldots, n)$ is the evidence from $\operatorname{PZTA}(j)$. The new BPA denoted by $m_{j}^{\prime}(A)$, as shown in (9), can be recalculated by the matrix $W$ to be

$$
m_{j}^{\prime}(A)= \begin{cases}w_{j} \cdot m_{j}(A), & A \subset \Theta \\ 1-\sum_{B \subset \Theta} w_{j} \cdot m_{j}(A), & B \subset \Theta, A=\Theta .\end{cases}
$$

The term $w_{j} \cdot m_{j}(A)$ in (9) indicates the BPA of $A \subset \Theta$ is reduced (as $\left.0<w_{j} \leq 1\right)$. And the term $1-\sum_{B C \Theta} w_{j} \cdot m_{j}(A)$ indicates the loss of BPA will be added to the BPA of $\Theta$. Based on the D-S evidence theory, the BPA for the hypothesis supported by less importance will be significantly reduced. By 
TABLE 1: Adjusted BPAs by the weighted index.

\begin{tabular}{lccc}
\hline & $\begin{array}{c}m\left(F_{1}\right) \\
\text { (weighted index) } \\
m^{\prime}\left(F_{1}\right)\end{array}$ & $\begin{array}{c}m\left(F_{2}\right) \\
\text { (weighted index) } \\
m^{\prime}\left(F_{2}\right)\end{array}$ & $\begin{array}{c}m(\Theta) \\
\text { (weighted index) } \\
m^{\prime}(\Theta)\end{array}$ \\
\hline$m_{1}$ & 0.8 & 0.2 & 0.0 \\
$m_{1}^{\prime}$ & $(0.9 / 0.9=1)$ & $(0.9 / 0.9=1)$ & $(0.9 / 0.9=1)$ \\
\hline$m_{2}$ & $0.8 * 1=0.8$ & $0.2 * 1=0.2$ & $1-0.8-0.2=0.0$ \\
$m_{2}^{\prime}$ & 0.1 & 0.9 & $(0.3 / 0.9=0.3333)$ \\
\hline
\end{tabular}

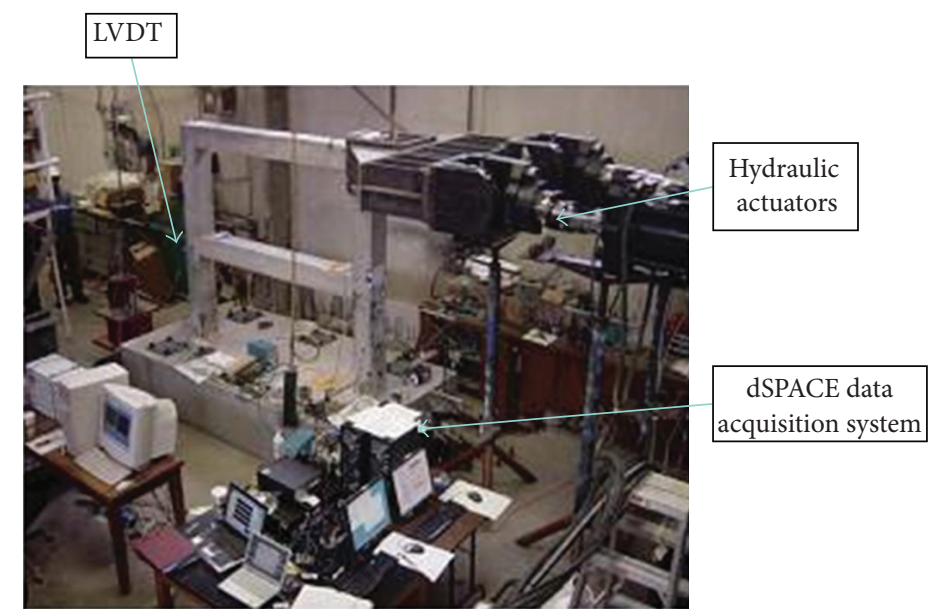

FIGURE 5: Concrete frame for the structural health monitoring test.

fusing the new BPA, the fusion result $m(A), A \in\left\{F_{1}, F_{2}, \Theta\right\}$, can be obtained by Dempster's combination rule shown in (5). The $m\left(F_{1}\right)$ can be regarded as the weighted fusion damage index.

The following example shows how the WFDI can be calculated. Assume that one PZTS obtains different data from two PZTAs. The RMSD can be calculated through the data, and then the BPAs are shown as follows:

$$
\begin{array}{lll}
m_{1}\left(F_{1}\right)=0.8, & m_{1}\left(F_{2}\right)=0.2, & m_{1}(\Theta)=0.0 . \\
m_{2}\left(F_{1}\right)=0.1, & m_{2}\left(F_{2}\right)=0.9, & m_{2}(\Theta)=0.0 .
\end{array}
$$

Assume that the first evidence importance is larger than the second evidence importance, or $v_{1}=0.9$, and $v_{2}=0.3$ (in reality, these can be calculated based on the distances between the PZTS and the two PZTAs). That means the first evidence is more reliable than the second.

The new BPAs are calculated and shown in Table 1. The losses of $m_{2}\left(F_{1}\right)$ and $m_{2}\left(F_{2}\right)$ are added to $m_{2}(\Theta)$. The second evidence's influence on the fusion result is reduced.

By using (5), the fusion results are obtained as follows:

$$
\begin{aligned}
K & =1-\sum_{A_{1} \cap \cdots \cap A_{n}=\emptyset} m_{1}\left(A_{1}\right) \cdots m_{n}\left(A_{n}\right) \\
& =1-(0.8 * 0.3+0.2 * 0.0333)=0.75334,
\end{aligned}
$$

$$
\begin{aligned}
m\left(F_{1}\right) & =\left(m_{1} \oplus m_{2}\right)\left(F_{1}\right) \\
= & \frac{1}{K} \sum_{A_{1} \cap A_{2}=F_{1}} m_{1}\left(A_{1}\right) \cdot m_{2}\left(A_{2}\right) \\
= & \frac{0.8 * 0.0333+0.8 * 0.6667}{0.75334}=0.7434, \\
m\left(F_{2}\right) & =\left(m_{1} \oplus m_{2}\right)\left(F_{2}\right) \\
& =\frac{1}{K} \sum_{A_{1} \cap A_{2}=F_{2}} m_{1}\left(A_{1}\right) \cdot m_{2}\left(A_{2}\right) \\
& =\frac{0.2 * 0.3+0.2 * 0.6667}{0.75334}=0.2566, \\
m(\Theta)=1- & m\left(F_{1}\right)-m\left(F_{2}\right)=1-0.7434-0.2566=0 .
\end{aligned}
$$

It is shown that the fusion result depends more on the first evidence $\left(m\left(F_{1}\right)=0.7434\right.$ is close to $m_{1}\left(F_{1}\right)=0.8$ as com-pared to $\left.m_{2}\left(F_{1}\right)=0.1\right) . m\left(F_{1}\right)=0.7434$ is the WFDI. It indicates that the structure is seriously damaged, which is a conclusion similar to the first evidence $\left(m_{1}\left(F_{1}\right)=0.8\right)$. The WFDI can avoid the disturbance of the second evidence, which is less reliable. In this paper, the developed weighted fusion damage will be used to quantitatively evaluate the health status and provide more precise monitoring results for a pushover test of a concrete frame. 


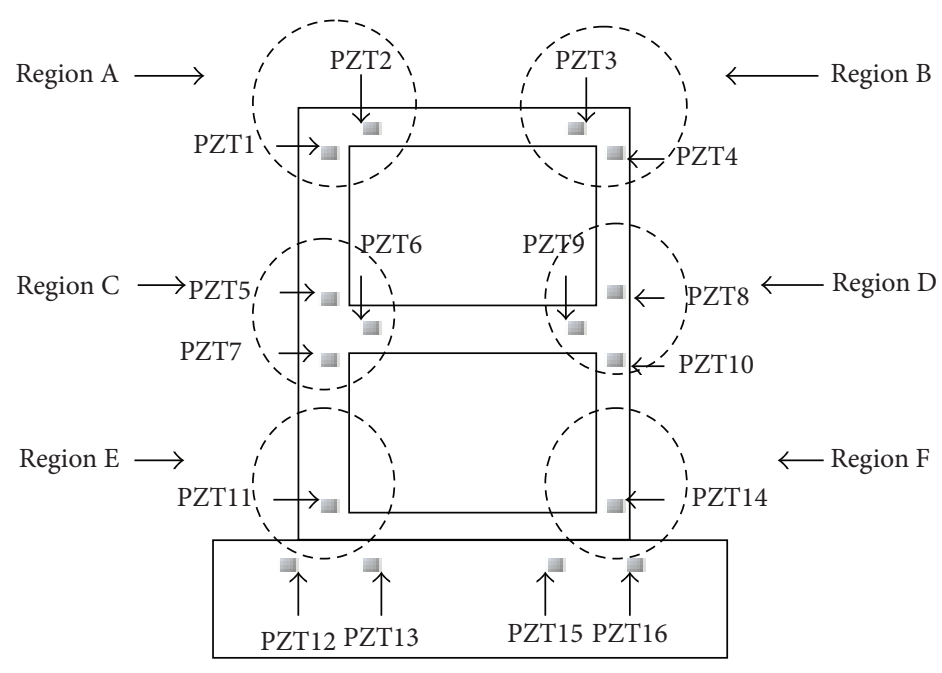

FigURE 6: Locations of the smart aggregates.

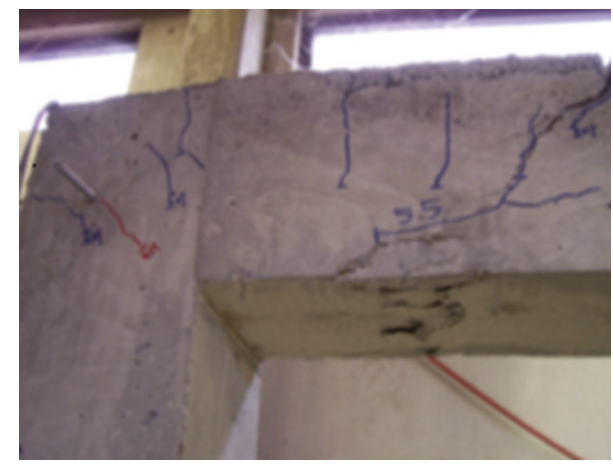

(a) Cracks at region $\mathrm{A}$

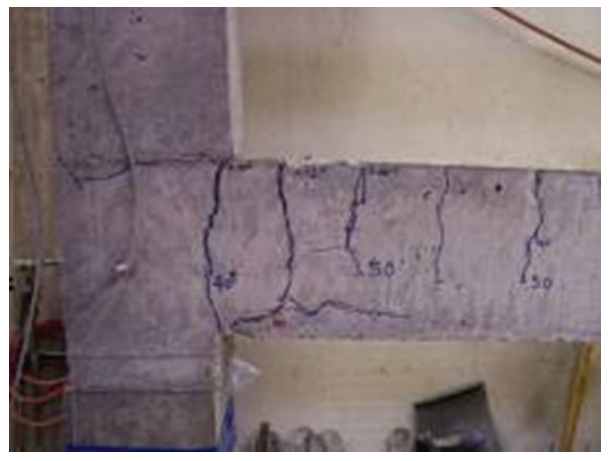

(c) Cracks at region $\mathrm{C}$



(e) Cracks at region $\mathrm{E}$

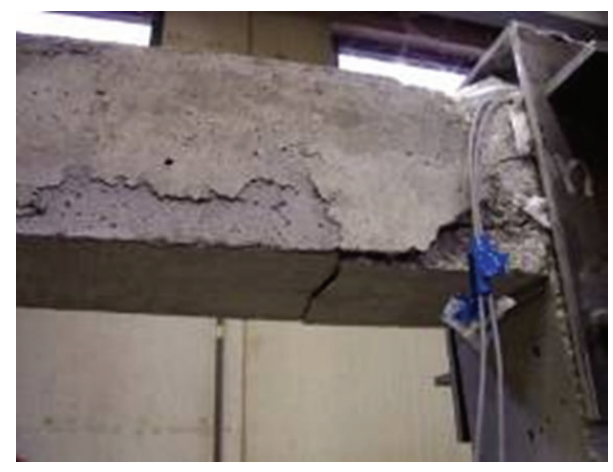

(b) Cracks at region $\mathrm{B}$



(d) Cracks at region $\mathrm{D}$

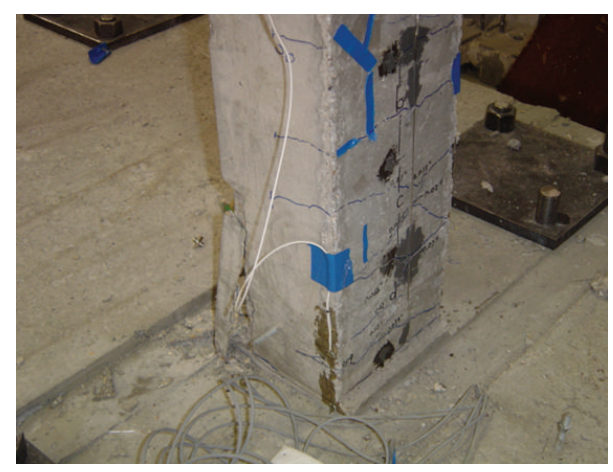

(f) Cracks at region $\mathrm{F}$

Figure 7: Damage status of concrete frame after failure. 




FIGURE 8: Crack width versus the load for a crack which first appears in the concrete frame.

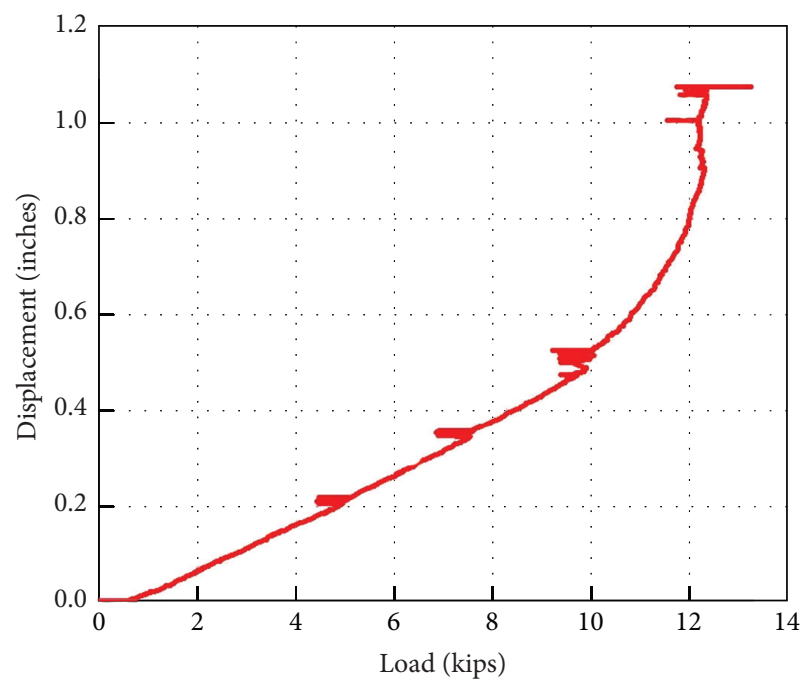

FIGURE 9: Displacement versus load.

\section{Test Frame and Instrumentation Details}

4.1. Experimental Program of Structural Health Monitoring. The data of structural health monitoring of a two-story concrete frame instrumented with piezoceramic-based smart aggregates, as shown in Figure $5[7,8]$, are used in this study. The locations of the smart aggregates are shown in Figure 6. Two hydraulic actuators were used to apply load to the frame structure at its right corner to conduct the so-called pushover test. The load was increasingly applied to the frame until its structural failure. Linear variable differential transformers (LVDTs) were used to measure the displacement at different locations of the concrete frame. In addition, hand-held microscopes were also used to measure the crack width.

During the push-over test, the active-sensing based structural health monitoring enabled by smart aggregates was implemented to evaluate the damage status of the concrete frame. Before the appearance of a major crack, the loading protocol was the force control mode. After the appearance of a major crack, the loading protocol was set to the displacement control mode. During the force control mode, the load (force) was increased gradually at a fixed rate until the major crack appears. During the displacement control mode, the concrete
TABLE 2: Details for each test during the loading process.

\begin{tabular}{lc}
\hline Test number & Description \\
\hline 1 & Health status \\
2 & Load $=4.41 \mathrm{kips}$ \\
3 & Load $=6.61 \mathrm{kips}$ \\
4 & Load $=8.82 \mathrm{kips}$ \\
5 & Load $=11.02 \mathrm{kips}$ \\
6 & Displacement $=1.6$ inches \\
7 & Displacement $=1.9$ inches \\
8 & Displacement $=1.9$ inches \\
9 & Displacement $=2.5$ inches \\
10 & Displacement $=3$ inches \\
11 & Displacement $=3$ inches \\
12 & Displacement $=3.5$ inches \\
13 & Displacement $=4$ inches \\
14 & Failed \\
\hline
\end{tabular}

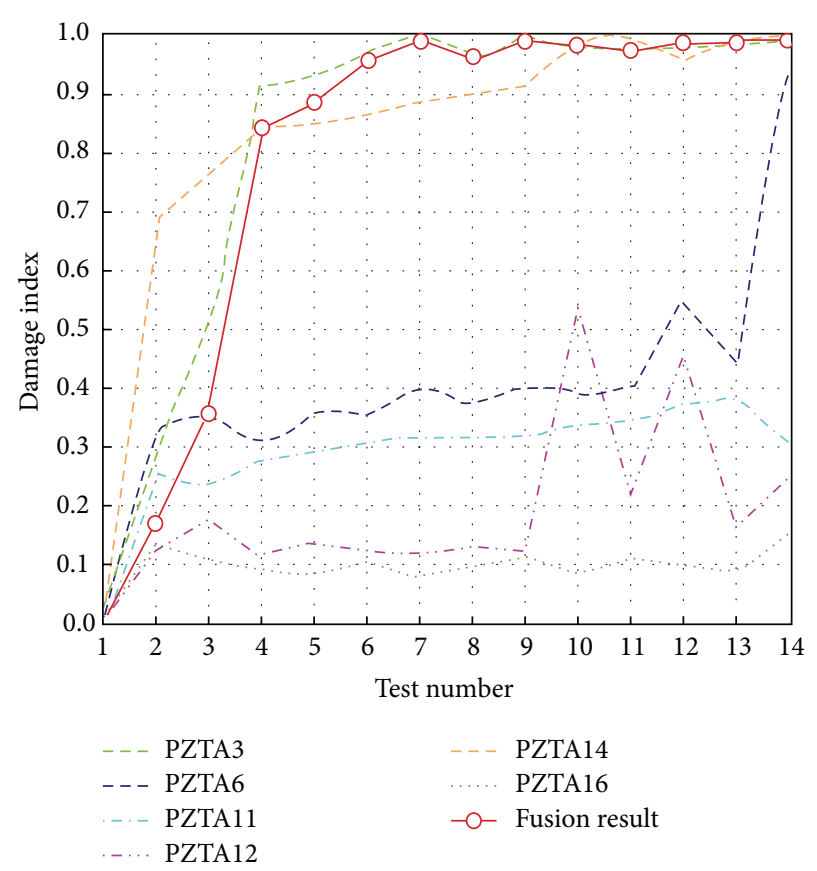

Figure 10: Damage index of PZTS2 with fusion result.

frame was pushed by the hydraulic actuators to a certain position at a specified rate and these positions were held for a certain time for data acquisition. During both load control and displacement control modes, the data of the active-sensing based structural health monitoring enabled by smart aggregates were recorded at different time intervals, which are designated as different test numbers. The detailed loading information at each structural health monitoring test is shown in Table 2. After the failure of the concrete frame, the damages at different locations are shown in Figure 7.

The width for the first crack was continuously measured by a hand-held microscope, and the data is plotted versus the load, as shown in Figure 8, which clearly reveals that the relationship between the crack width and the load value is close to a linear one before the load of 11.02 kips. After the load value 


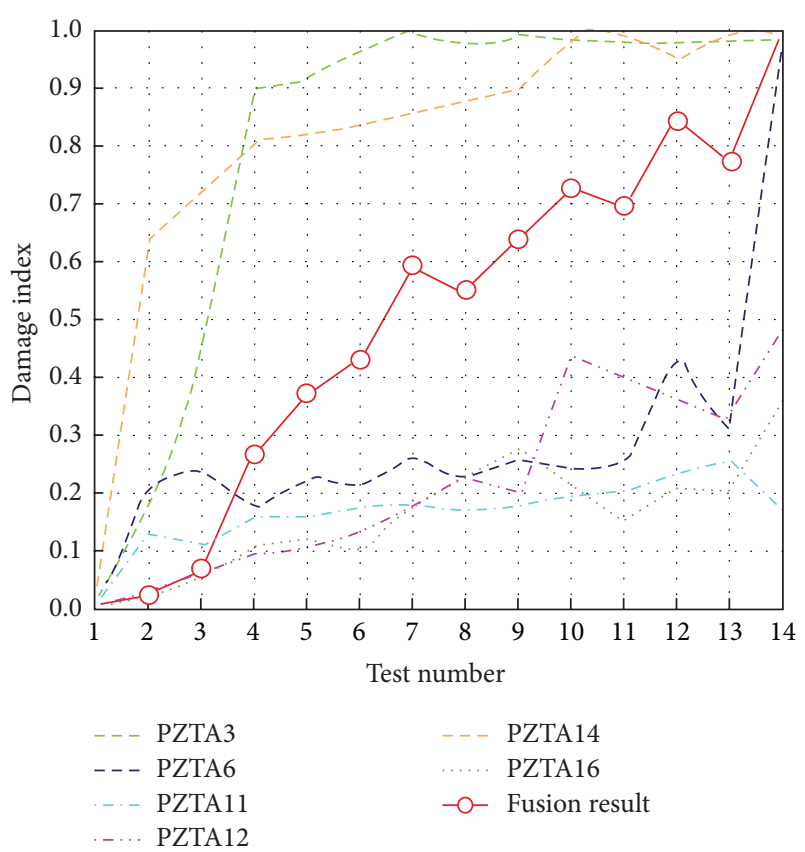

FIGURE 11: Damage index of PZTS8 with fusion result.

reaches 11.02 kips, the crack width increases dramatically due to yielding of the reinforcing steel bars in the concrete frame. In Figure 9, the results of the LVDT measurements show that the relationship between displacement and load was highly nonlinear after the load value reached 11.02 kips. This LVDT was positioned at the top of the concrete frame. The LVDT data also verified that the concrete frame yielded at a load value of 11.02 kips.

\subsection{Structural Health Monitoring Using the Weighted Fusion} Damage Index. During the active-sensing health monitoring experiment, PZT3, PZT6, PZT11, PZT12, PZT14, and PZT16 were alternately used as actuators. When one smart aggregate was chosen as an actuator (PZTA) to generate sweep sine waves, the other smart aggregates were used as sensors (PZTSs). The PZTSs detected the sweep sine responses. To obtain more reliable results, the developed WFDI was used to process multiple datasets with different smart aggregates. The damage indices were calculated by (1) from the data of PZTSs and the weighted index was calculated by (6) and (7). The BPA in the D-S evidence theory can be obtained from the damage index and adjusted by the weighted index. Then the WFDI can be calculated using (5).

As illustrated in Figure 10, the solid line is the WFDI based on the PZTS2's data. The other dashed lines represent the damage indices without using data fusion when PZT2 was used as the sensor and each of the other smart aggregates was alternatively used as an actuator. Figures 11 and 12 show the WFDI results of PZTS8 and PZTS15. Comparing among WFDI results of PZTS2, PZTS8, and PZTS15, the PZTS2's WFDI has the largest fusion value, PZTS15 has the lowest fusion value, and PZTS8 has the medium fusion value. These results show that among PZT2, PZT8, and PZT15, the area around PZT2 had the most severe damage and the area

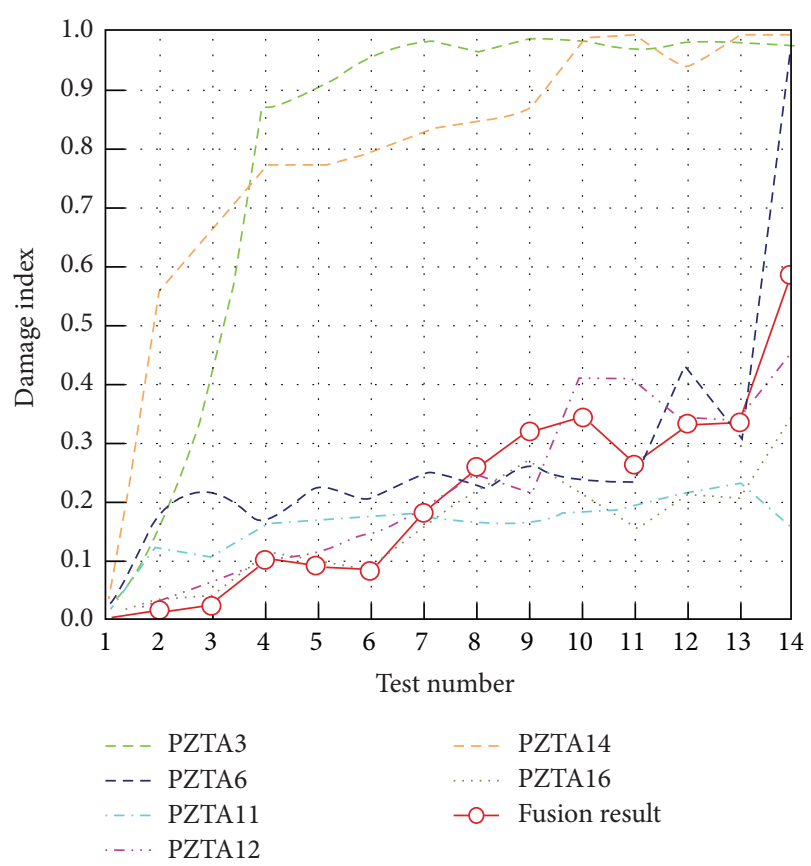

Figure 12: Damage index of PZTS15 with fusion result.

around PZT15 had the least severe damage, which are all confirmed by visual inspection of the specimen. PZT15 was embedded inside the base where damage was less likely to happen.

From the WFDI comparison results shown in Figure 13, PZTS2, PZTS6, and PZTS14 have much higher WFDI values than PZT9S and PZT11S. This demonstrates that the damage statuses around PZT2, PZT6, and PZT14 were more severe than those around PZT9 and PZT11, which was verified by the visual inspection results shown in Figure 7. Additionally, the yielding point obtained from the WFDI results was verified by the hand-held microscope data shown in Figure 8 and the LVDT data shown in Figure 9. As shown in Figures 8 and 9, the structure yielded when the load was over 11.02 kips. From Table 2, the load of 11.02 kips corresponds to the test number 5. From the WFDI results shown in Figure 13, the prediction point (WFDI is over 0.9) for yielding is at test number 3, which corresponds to the 6.61-kip load value. The proposed fusion damage index is more sensitive in predicting yielding point than the observation based on measurements from the microscope and the LVDT.

Figure 14 shows the comparison between the WFDI results and the displacement of the top part of the structure when the load was increasingly applied to the structure until failure. PZTS2 and PZTS6 were selected for the comparison as they were located in the most seriously damaged and structurally important parts of the structure. As shown in the figure, the fusion damage indices are consistent with the structure's displacement-load relationship and are more sensitive than the LVDT measured displacement as fusion damage indices reach saturation much earlier than the displacement. By comparing the WFDI results and the load-displacement curve, it can be seen that the proposed weighted fusion damage index quantitatively evaluates the damage status 




FIgURE 13: Weighted fusion damage index versus damage status at different locations.

during the loading process. Furthermore, the experimental results verify that proposed WFDI is more sensitive in detecting the yielding point than the traditional displacement-load curve. From the traditional displacement-load curve shown in Figure 14, the curve becomes nonlinear when the load was 11.2 kips. This shows that the frame yielded at a load value of 11.2 kips. From the WFDI results shown in Figure 14, the WFDI curve of PZTS2 reached a critical value around a load value of 6.5 kips and the WFDI curve of PZTS6 reached a critical value at a load value of 8.5 kips. The critical point detected by WFDI was ahead of the yielding point detected by traditional displacement-load curve which experimentally verified the effectiveness and the sensitiveness of the proposed WFDI.

\section{Conclusions}

In this paper, an innovative data fusion-based structural health monitoring approach is proposed to provide accurate and comprehensive health monitoring results. In the proposed approach, a weighted fusion damage index is developed based on the D-S evidence theory and the wavelet packet analysis. Structural health monitoring data of a twostory concrete frame instrumented with smart aggregates were used for this study. The weighted fusion damage indices were developed and applied to analyze and interpret the experimental data. Analyses show that the proposed weighted fusion damage index can reveal the damage status of different areas of the frame. The results are consistent with the visual inspection of the cracks on the concrete frame. Furthermore, analyses also demonstrate that the weighted fusion damage index not only evaluates the damage severity but also is more sensitive than traditional health monitoring approaches that

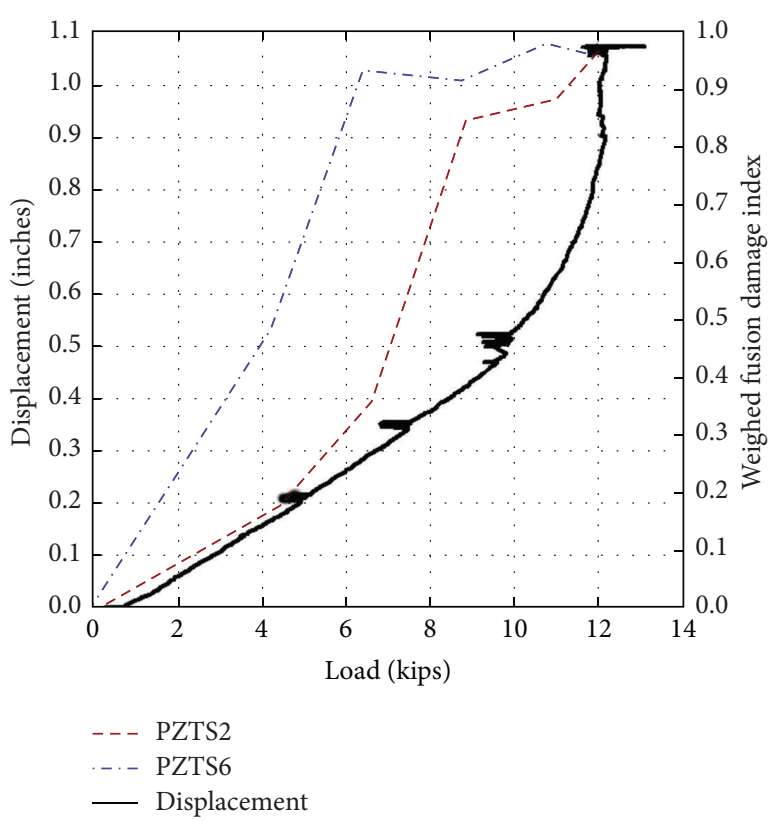

FIGURE 14: Weighted fusion damage index and displacement versus load.

use hand-held microscopes or LVDTs. In addition, the damage location information can be extracted by the proposed approach.

\section{Conflict of Interests}

The authors declare that there is no conflict of interests regarding the publication of this paper. 


\section{Acknowledgments}

This work was partially funded by the National Science Foundation (NSF) via Research Grants (nos. CMMI-0724190 and CNS-0832089), the National Natural Science Foundation of China (no. 41104106, no. 51278387, and no. 51278084), and the Science Fund for Creative Research Groups Grant no. 51121005. The opinions expressed in this study are those of the authors and do not necessarily reflect the views of the sponsor.

\section{References}

[1] C. K. Soh, K. K.-H. Tseng, S. Bhalla, and A. Gupta, "Performance of smart piezoceramic patches in health monitoring of a RC bridge," Smart Materials and Structures, vol. 9, no. 4, pp. 533$542,2000$.

[2] A. S. K. Naidu and C. K. Soh, "Identifying damage location with admittance signatures of smart piezo-transducers," Journal of Intelligent Material Systems and Structures, vol. 15, no. 8, pp. 627$642,2004$.

[3] H. Fu and Q. Xu, "Locating impact on structural plate using principal component analysis and support vector machines," Mathematical Problems in Engineering, vol. 2013, Article ID 352149, 8 pages, 2013.

[4] Z. Kral, W. Horn, and J. Steck, "Crack propagation analysis using acoustic emission sensors for structural health monitoring systems," The Scientific World Journal, vol. 2013, Article ID 823603, 13 pages, 2013.

[5] J.-B. Ihn and F.-K. Chang, "Pitch-catch active sensing methods in structural health monitoring for aircraft structures," Structural Health Monitoring, vol. 7, no. 1, pp. 5-19, 2008.

[6] G. Song, H. Gu, Y. L. Mo, T. T. C. Hsu, and H. Dhonde, "Concrete structural health monitoring using embedded piezoceramic transducers," Smart Materials and Structures, vol. 16, no. 4, pp. 959-968, 2007.

[7] G. Song, H. Gu, and Y.-L. Mo, "Smart aggregates: multi-functional sensors for concrete structures-a tutorial and a review," Smart Materials and Structures, vol. 17, no. 3, Article ID 033001, 2008.

[8] A. Laskar, H. Gu, Y. L. Mo, and G. Song, "Progressive collapse of a two-story reinforced concrete frame with embedded smart aggregates," Smart Materials and Structures, vol. 18, no. 7, Article ID 075001, 2009.

[9] L. Yu, V. Giurgiutiu, J. Wang, and Y.-J. Shin, "Corrosion detection with piezoelectric wafer active sensors using pitch-catch waves and cross-time-frequency analysis," Structural Health Monitoring, vol. 11, no. 1, pp. 83-93, 2012.

[10] Z. Sun, B. Rocha, K. T. Wu, and N. Mrad, "A Methodological review of piezoelectric based acoustic wave generation and detection techniques for structural health monitoring," International Journal of Aerospace Engineering, vol. 2013, Article ID 928627, 22 pages, 2013.

[11] Y. Q. Ni, X. W. Ye, and J. M. Ko, "Monitoring-based fatigue reliability assessment of steel bridges: analytical model and application," Journal of Structural Engineering, vol. 136, no. 12, pp. 1563-1573, 2010.

[12] Y. Q. Ni, X. W. Ye, and J. M. Ko, "Modeling of stress spectrum using long-term monitoring data and finite mixture distributions," Journal of Engineering Mechanics, vol. 138, no. 2, pp. 175183, 2011.
[13] H. Guo and L. Zhang, "A weighted balance evidence theory for structural multiple damage localization," Computer Methods in Applied Mechanics and Engineering, vol. 195, no. 44-47, pp. 6225-6238, 2006.

[14] H. Y. Guo, "Structural damage detection using information fusion technique," Mechanical Systems and Signal Processing, vol. 20, no. 5, pp. 1173-1188, 2006.

[15] P. A. Vanniamparambil, I. Bartoli, K. Hazeli et al., "An integrated structural health monitoring approach for crack growth monitoring," Journal of Intelligent Material Systems and Structures, vol. 23, no. 14, pp. 1563-1573, 2012.

[16] Y. Bao and H. Li, "Application of information fusion and Shannon entropy in structural damage detection," in Health Monitoring of Structural and Biological Systems, Proceedings of SPIE, San Diego, Calif, USA, March 2007.

[17] X. Fan and M. J. Zuo, "Fault diagnosis of machines based on D-S evidence theory. Part 1: D-S evidence theory and its improvement," Pattern Recognition Letters, vol. 27, no. 5, pp. 366376, 2006.

[18] H. Y. Guo and Z. L. Li, "A two-stage method to identify structural damage sites and extents by using evidence theory and micro-search genetic algorithm," Mechanical Systems and Signal Processing, vol. 23, no. 3, pp. 769-782, 2009.

[19] S. Thenozhi, W. Yu, A. L. López Chau, and X. Li, "Structural health monitoring of tall buildings with numerical integrator and convex-concave hull classification," Mathematical Problems in Engineering, vol. 2012, Article ID 212369, 15 pages, 2012.

[20] X. Zhao, M. Li, G. Song, and J. Xu, "Hierarchical ensemblebased data fusion for structural health monitoring," Smart Materials and Structures, vol. 19, no. 4, Article ID 045009, 2010.

[21] K. K.-H. Tseng and A. S. K. Naidu, "Non-parametric damage detection and characterization using smart piezoceramic material," Smart Materials and Structures, vol. 11, no. 3, pp. 317-329, 2002.

[22] G. Park, H. Sohn, C. R. Farrar, and D. J. Inman, "Overview of piezoelectric impedance-based health monitoring and path forward," The Shock and Vibration Digest, vol. 35, no. 6, pp. 451463, 2003.

[23] A. P. Dempster, "A generalization of Bayesian inference," Journal of the Royal Statistical Society B, vol. 30, pp. 205-247, 1968.

[24] G. Shafer, A Mathematical Theory of Evidence, Princeton University Press, Princeton, NJ, USA, 1976.

[25] R. R. Yager and L. Liu, Classic Works of the Dempster-Shafer Theory of Belief Functions, Springer, New York, NY, USA, 2008. 


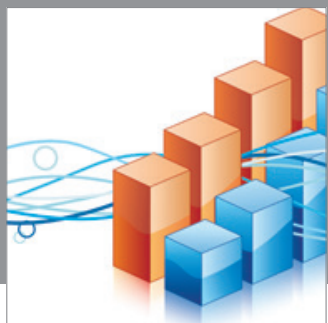

Advances in

Operations Research

mansans

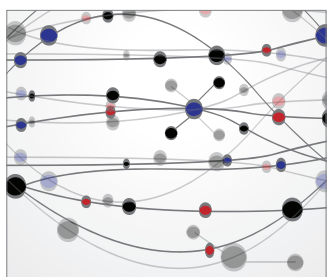

The Scientific World Journal

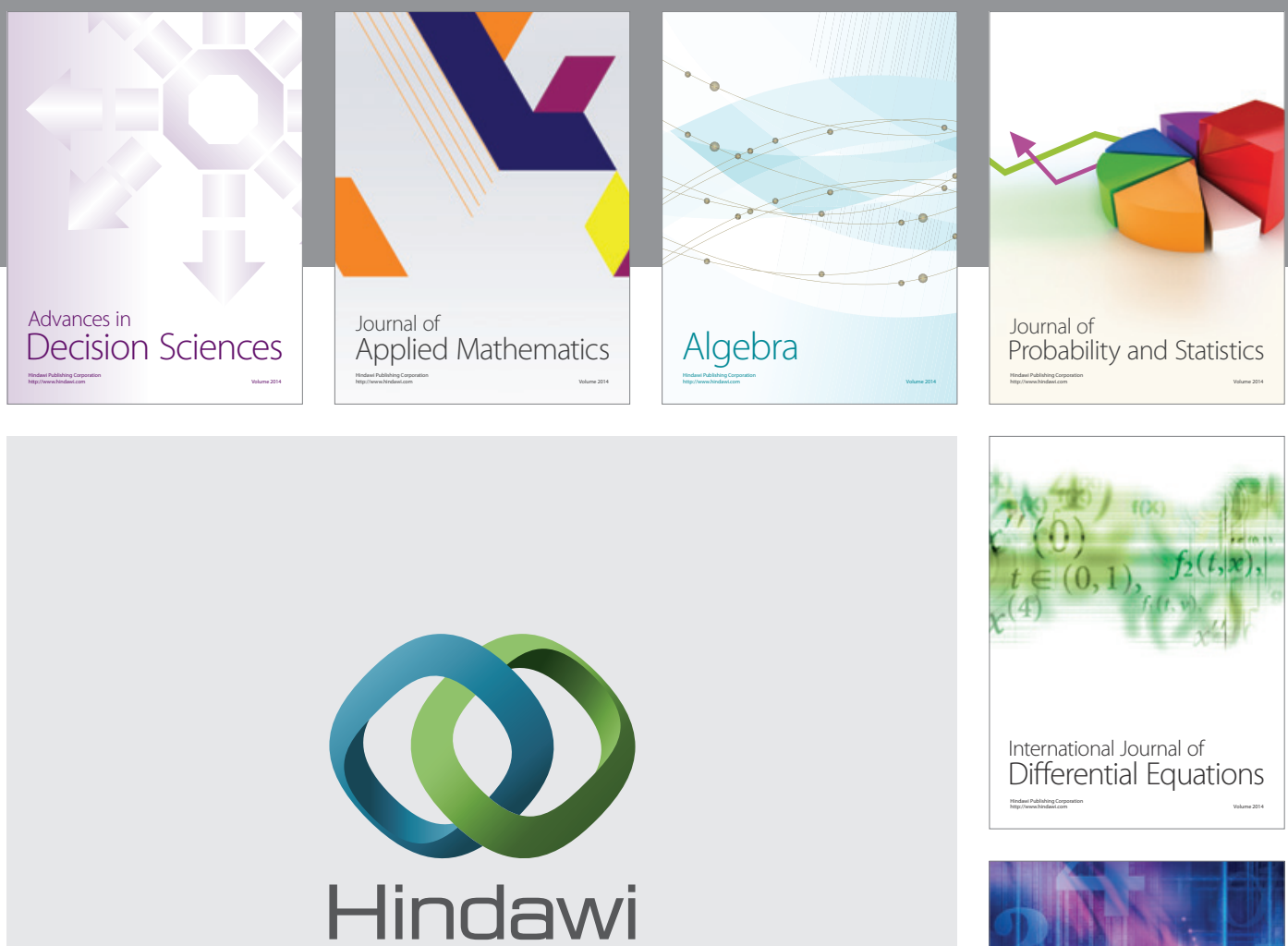

Submit your manuscripts at http://www.hindawi.com
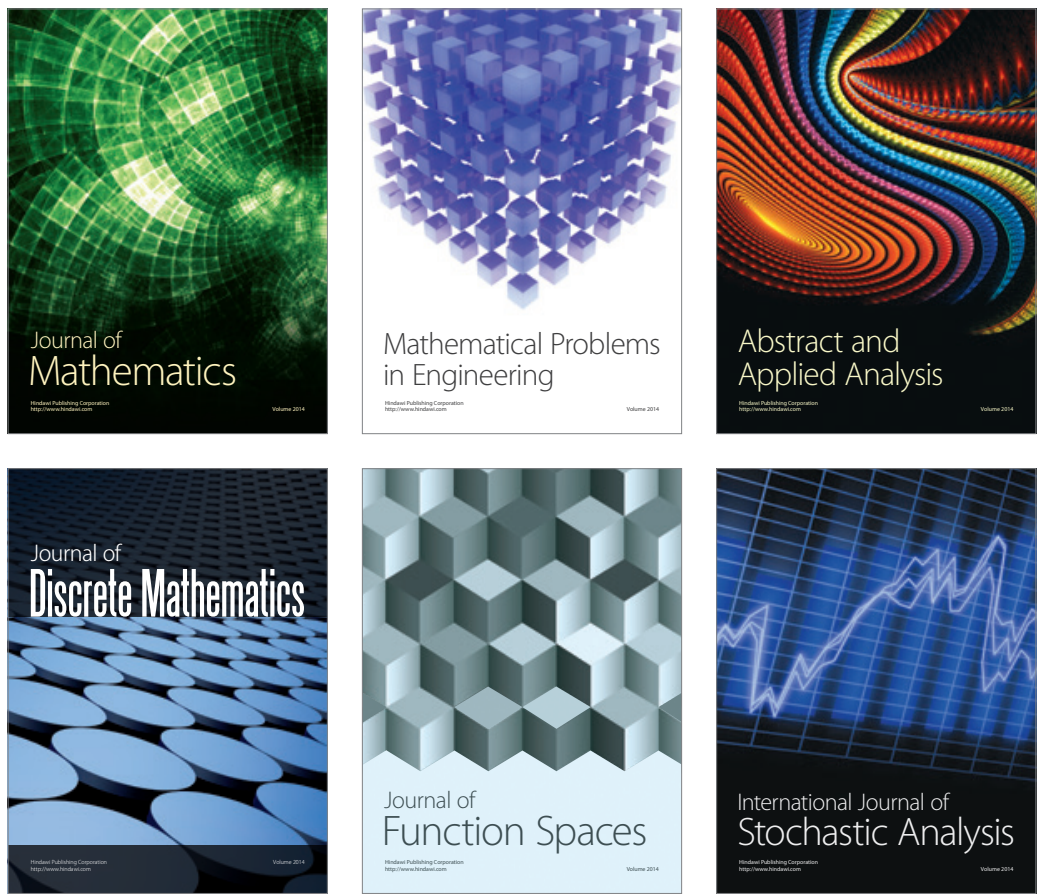

Journal of

Function Spaces

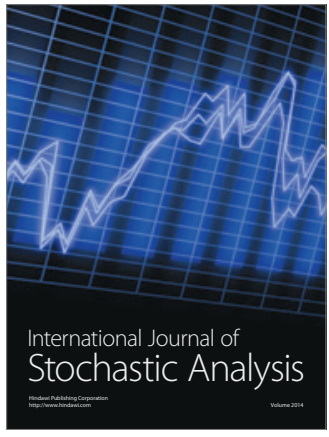

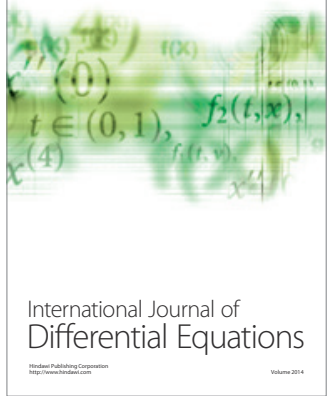
